\title{
Referral steps in district health system are side-stepped
}

\author{
M E Mojaki, D Basu, M E Letskokgohka, M Govender
}

To the Editor: The South African public health sector follows a hierarchical referral system. District hospitals play a central role between the primary health care (PHC) clinics, community health centres (CHCs), regional and tertiary hospitals. They provide level one (generalist) services to in- and outpatients referred from PHC clinics and CHCs. District hospitals should ensure that patients are treated at the appropriate level of care and receive continuity of care. ${ }^{1}$ However, patients often go directly to district hospitals, resulting in increased caseloads. Studies have identified several factors (e.g. accessibility, acceptability, efficiency and effectiveness) that might influence a referral system and its usage. ${ }^{2-4}$ We aimed to develop an understanding of the South African district referral system.

\section{Methods}

A cross-sectional study design was applied at the Dr J S Moroka Hospital, the only district hospital in the Thaba Nchu health subdistrict, Free State, and a referral point for 11 clinics in the sub-district, 4 clinics from the neighbouring sub-district, and 5 GPs' rooms. Routinely collected information on patients' records and registers was reviewed. The study was approved by the Human Research Ethics Committee (Medical) of the University of the Witwatersrand.

\section{Results}

A total of 528 patients were registered during the study period; 241 (46\%) were registered in the out-patient department (OPD) and 287 (54\%) in the casualty department. Categorising these patients suggested that $68 \%$ in OPD and $38 \%$ in casualty units would require hospital care; the rest could be seen at the PHC facilities (clinic and $\mathrm{CHC}$ ). One hundred and twelve patients (39\%) in casualty were seen during weekends. More than $50 \%$ of patients in both units were self-referrals. Ambulances took $31 \%$ of the patients directly to the hospital's casualty department, bypassing the nearest PHC facilities.

Although $66 \%$ of chronic cases were seen in OPD, a third of them came to casualty; $28 \%$ of patients seen after-hours at casualty were chronic cases. In both designated areas, most patients were treated and discharged on the same day. As expected, most patients (68) (25\%) were admitted from casualty rather than OPD (18) (10\%).

Most acute cases (75\%) were seen at casualty; of these, $60 \%$ were self-referred, whereas most (82) (61\%) chronic patients were referred. The proportion of self-referred patients was similar (52\%) during working hours and after hours. During weekends, $56 \%$ of patients were self-referrals, as 9 out of 11 clinics do not operate after hours.

In the OPD, the most common presenting problems were circulatory system related (35) (17\%) followed by infectious and

Dr J S Moroka District Hospital, Thaba Nchu, Free State Province, and School of Public Health, University of the Witwatersrand, Johannesburg M E Mojaki, MPH (Hosp Mgmt)

Department of Community Health, Charlotte Maxeke Johannesburg Hospital and University of the Witwatersrand

D Basu, MB BS, MSc (Med), MPH, MBA, MMed, FCPHM, PhD (Med)

School of Public Health, University of the Witwatersrand M E Letskokgohka, MPH (Hosp Mgmt)

M Govender, MB ChB, MSc parasitic diseases (24) (12\%), injury and poisoning (23) (11\%) and respiratory diseases (18) (9\%). In casualty, most patients were diagnosed with injury and poisoning $(76)(28 \%)$, respiratory diseases (46) (17\%), circulatory system diseases (26) (10\%) and diseases of the digestive system (31) (11\%). Commonly cited reasons for self-referral were desire to be seen by doctors (47\%) and perceived poor services at clinics (32\%). The average estimated distance travelled by patients to their nearest clinic was $11 \mathrm{~km}$ (range $3-40 \mathrm{~km}$ ); viz. the $24 \mathrm{~km}$ to this hospital (range $14-56 \mathrm{~km}$ ).

\section{Discussion}

Most patients seen in OPD and casualty had bypassed the referral system. More than half of the patients seen at these units could have been managed at the PHC facilities, which is similar to findings at King Edward VIII Hospital in Durban. ${ }^{5}$ Many patients were brought straight to the hospital by ambulance, although $25 \%$ of them did not require hospital care. Research is needed on assessment criteria used by ambulance personnel for bringing patients to health facilities.

We attribute overburdening of district hospitals to failure of the PHC facilities to attract their catchment population. Long waiting times at this hospital's casualty (median 329 minutes) could easily be reduced if these patients were managed at their nearest PHC facilities. Most acute cases at the hospital were self-referral where the patients probably did not seek medical care until they became severely ill or sought care at other levels of health care, and then came to the hospital.

The reasons for patients bypassing PHC facilities may include their perception of superior care and resource availability in hospitals, desire to be seen by medical doctors, and that a hospital may be the nearest health facility. ${ }^{1}$ Other cited reasons were clinic hours (most operate 8 hours a day for 5 days), dysfunctional CHCs, lack of public transport, and lack of education about the referral system among patients and health professionals. Few patients $(2 \%)$ were educated on the referral system, and none had been charged bypass fees, despite a provincial policy for implementing these. Patients' tendency to bypass PHC facilities distorts core functions of hospitals and is probably linked to overcrowded OPD and casualty in these hospitals. PHC provision by hospitals is uneconomical, as treatment cost per illness is much more expensive than at PHC facilities. ${ }^{3}$

\section{Conclusion}

Evidence is provided of an ineffective referral system in a district hospital in South Africa. Regular visits by hospital doctors to PHC facilities, flexible and longer operating hours of clinics, functional CHCs, regular supplies of medication, and education of patients and health professionals about the referral system can improve the system and provide better patient care within the district health system.

\section{References}

1. Engelbrecht B, Daviaud E, Shaw V, Couper I, Classens L. In: Baron P, McCoy D, eds. A Barometer of District Hospital Management. Durban: Health Systems Trust, 2000: 27.

2. Hensher M, Price M, Adomakoh S. Referral hospitals. In: Jamison DT, Breman JG, Measham AR, et al., eds. Disease Control Priorities in Developing Countries. 2nd ed. Washington (DC): World Bank, 2006: 1229-1242. 3. Akande TM. Referral systems in Nigeria: Study of a tertiary health facility. Annals of African Medicine 2004;3(3):130-133.

4. Middleton KR, Hing E. National hospital ambulatory medical care survey: 2004 outpatient departmen summary. CDC Advance Data 2006;373(3):130-133.

5. Rutkove SB, Abdool Karim SS, Loening WEK. Patterns of care in an overburdened tertiary hospital outpatients department. S Afr Med J 1990;77:476-478. 